\title{
Chiral corrections to kaon-nucleon scattering lengths
}

\author{
N. Kaiser \\ Physik Department T39, Technische Universität München, \\ D-85747 Garching, Germany
}

\begin{abstract}
We calculate the threshold T-matrices of kaon-nucleon and antikaon-nucleon scattering to one loop order in $\mathrm{SU}(3)$ heavy baryon chiral perturbation theory. To that order the complex-valued isospin-1 $\bar{K} N$ threshold T-matrix can be successfully predicted from the isospin-0 and $1 \mathrm{KN}$ threshold T-matrices. As expected perturbation theory fails to explain the isospin- $0 \bar{K} N$ threshold T-matrix which is completely dominated by the nearby subthreshold $\Lambda^{*}(1405)$-resonance. Cancelations of large terms of second and third chiral order are observed as they seem to be typical for $\mathrm{SU}(3)$ baryon chiral perturbation theory calculations. We also give the kaon and eta loop corrections to the $\pi N$ scattering lengths and we investigate $\pi \Lambda$ scattering to one-loop order. The second order s-wave low-energy constants are all of natural size and do not exceed $1 \mathrm{GeV}^{-1}$ in magnitude.
\end{abstract}

PACS: 12.38.Bx, 12.39.Fe, 13.75.Gx, 13.75.Jz

To be published in: Physical Review $C$.

Chiral symmetry severely constrains the interaction of the strongly interacting particles at low energies, first formulated in terms of current algebra. One of the most splendid successes of current algebra was the Tomozawa-Weinberg prediction [1] for the s-wave pion-nucleon scattering lengths $a_{\pi N}^{ \pm}$. The tool to systematically investigate the consequences of spontaneous and explicit chiral symmetry breaking in QCD is chiral perturbation theory. Observables are calculated with the help of an effective field theory formulated in terms of the Goldstone bosons $(\pi, K, \bar{K}, \eta)$ and the low-lying baryons $(N, \Lambda, \Sigma, \Xi)$. A systematic expansion in small external momenta and meson masses is possible. Pion-loop corrections to $a_{\pi N}^{ \pm}$have been first calculated in ref.[2]. In the case of the isospin-odd $\pi N$-scattering length $a_{\pi N}^{-}$the chiral loop correction in fact closes the about $15 \%$ gap between the lowest order Tomozawa-Weinberg prediction and the empirical value [3]. Recently, the complete fourth order one-loop analysis of low energy $\pi N$-scattering has been performed in ref.[4].

Kaon-nucleon and antikaon-nucleon scattering are then of interest as a testing ground of the three-flavor chiral dynamics. In comparison to the $\mathrm{SU}(2)$ sector of $\pi N$-interaction these processes involve several new complications. First, the pertinent expansion parameter is much larger since the pion mass gets replaced by the kaon mass. Secondly, for $\bar{K} N$-scattering there exist inelastic channels involving a pion and a hyperon down to threshold, while $K N$-scattering is purely elastic at low energies. The dynamical differences in $K N$ versus $\bar{K} N$-scattering naturally show up in the values of the s-wave scattering lengths or the equivalent threshold T-matrices, $T_{K N}^{(0,1)}=4 \pi\left(1+m_{K} / M_{N}\right) a_{K N}^{(0,1)}$ and $T_{\bar{K} N}^{(0,1)}=4 \pi\left(1+m_{K} / M_{N}\right) a_{\bar{K} N}^{(0,1)}$. The superscripts denote here 
the total isospin, $I=0$ or $I=1$. In a combined dispersion relation analysis of $K N$ and $\bar{K} N$ scattering data the following values for the threshold T-matrices have been obtained by Martin $[5]$

$$
\begin{gathered}
T_{K N}^{(0)}=0.4 \mathrm{fm}, \quad T_{K N}^{(1)}=-6.3 \mathrm{fm}, \\
T_{\bar{K} N}^{(0)}=(-32.6+13.0 i) \mathrm{fm}, \quad T_{\bar{K} N}^{(1)}=(7.1+11.5 i) \mathrm{fm} .
\end{gathered}
$$

A compilation of values from other determinations can be found in [6]. We consider the results from the dispersion relation analysis most reliable and therefore compare the chiral calculation only with the empirical values given in eqs. $(1,2)$.

Let us begin with recalling the effective $\mathrm{SU}(3)$ chiral Lagrangians for meson-meson and meson-baryon interaction, which read at leading order,

$$
\begin{gathered}
\mathcal{L}_{\phi \phi}^{(2)}=f^{2} \operatorname{tr}\left(u_{\mu} u^{\mu}+\frac{\chi_{+}}{4}\right), \\
\mathcal{L}_{\phi B}^{(1)}=\operatorname{tr}\left(\bar{B}\left(i \partial_{0} B+\left[\Gamma_{0}, B\right]\right)\right)-D \operatorname{tr}(\bar{B}\{\vec{\sigma} \cdot \vec{u}, B\})-F \operatorname{tr}(\bar{B}[\vec{\sigma} \cdot \vec{u}, B]) .
\end{gathered}
$$

Here, the su(3)-matrix $B$ represents the octet baryon fields $(N, \Lambda, \Sigma, \Xi)$. The chiral connection $\Gamma^{\mu}=\frac{i}{2}\left[\xi^{\dagger}, \partial^{\mu} \xi\right]$ and the axial vector quantity $u^{\mu}=\frac{i}{2}\left\{\xi^{\dagger}, \partial^{\mu} \xi\right\}$ generate interaction terms with the Goldstone bosons $(\pi, K, \bar{K}, \eta)$. These Goldstone boson fields are collected in a $\mathrm{SU}(3)$-matrix $\xi=\exp (i \phi / 2 f)$. The parameter $f$ is the weak meson decay constant in the $\mathrm{SU}(3)$ chiral limit. Furthermore, $D$ and $F$ in eq.(4) denote the $\mathrm{SU}(3)$ axial vector coupling constants of the baryons and $\vec{\sigma}$ is the usual Pauli spin-vector. Explicit chiral symmetry breaking is introduced via the quantity $\chi_{+}=\xi^{\dagger} \chi \xi+\xi \chi \xi^{\dagger}$ whose vacuum expectation value $2 \chi=2 \operatorname{diag}\left(m_{\pi}^{2}, m_{\pi}^{2}, 2 m_{K}^{2}-m_{\pi}^{2}\right)$ can be expressed in terms of the pion and kaon masses squared. At next-to-leading order the part of the effective chiral Lagrangian relevant for s-wave meson-baryon interaction reads,

$$
\begin{aligned}
\mathcal{L}_{\phi B}^{(2)}= & b_{D} \operatorname{tr}\left(\bar{B}\left\{\chi_{+}, B\right\}\right)+b_{F} \operatorname{tr}\left(\bar{B}\left[\chi_{+}, B\right]\right)+b_{0} \operatorname{tr}(\bar{B} B) \operatorname{tr}\left(\chi_{+}\right) \\
& +\left(2 d_{D}+\frac{D^{2}-3 F^{2}}{2 M_{0}}\right) \operatorname{tr}\left(\bar{B}\left\{u_{0}^{2}, B\right\}\right)+\left(2 d_{F}-\frac{D F}{M_{0}}\right) \operatorname{tr}\left(\bar{B}\left[u_{0}^{2}, B\right]\right) \\
& +\left(2 d_{0}+\frac{F^{2}-D^{2}}{2 M_{0}}\right) \operatorname{tr}(\bar{B} B) \operatorname{tr}\left(u_{0}^{2}\right)+\left(2 d_{1}+\frac{3 F^{2}-D^{2}}{3 M_{0}}\right) \operatorname{tr}\left(\bar{B} u_{0}\right) \operatorname{tr}\left(u_{0} B\right) .
\end{aligned}
$$

The first three terms proportional to the low-energy constants $b_{D, F, 0}$ are chiral symmetry breaking ones and thus contribute to the mass splittings in the baryon octet. The remaining doublederivative terms are accompanied by low-energy constants $d_{D, F, 0,1}$ plus $1 / M_{0}$-corrections, where $M_{0}$ is the baryon mass in the chiral limit. In the heavy baryon formalism used here such second order terms with fixed coefficients stem from the $1 / M_{0}$-expansion [7] of the original relativistic leading order Lagrangian $\mathcal{L}_{\phi B}^{(1)}$.

Now, we turn to the chiral expansion of the $K N$ and $\bar{K} N$ scattering threshold T-matrices. At leading order $\mathcal{O}(q)$ one simply has the analog of the Tomozawa-Weinberg relation for kaons and antikaons,

$$
T_{K N}^{(0)}=0, \quad T_{K N}^{(1)}=-\frac{m_{K}}{f_{K}^{2}}, \quad T_{\bar{K} N}^{(0)}=\frac{3 m_{K}}{2 f_{K}^{2}}, \quad T_{\bar{K} N}^{(1)}=\frac{m_{K}}{2 f_{K}^{2}} .
$$

We have written these contributions with the physical kaon decay constant $f_{K}$ instead with $f$ (the chiral limit value). Therefore eq.(6) subsumes already all those one-loop corrections at $\mathcal{O}\left(q^{3}\right)$ which renormalize $f$ to $f_{K}$. At next-to-leading order $\mathcal{O}\left(q^{2}\right)$ one has the tree-level 
contributions from the effective Lagrangian $\mathcal{L}_{\phi B}^{(2)}$. These contributions can be compactly written in the form,

$$
T_{K N}^{(0)}=\frac{m_{K}^{2}}{f_{K}^{2}} C_{0}, \quad T_{K N}^{(1)}=\frac{m_{K}^{2}}{f_{K}^{2}} C_{1}, \quad T_{\bar{K} N}^{(0)}=\frac{m_{K}^{2}}{2 f_{K}^{2}}\left(3 C_{1}-C_{0}\right), \quad T_{\bar{K} N}^{(1)}=\frac{m_{K}^{2}}{2 f_{K}^{2}}\left(C_{0}+C_{1}\right),
$$

when introducing the two combinations of low-energy constants $C_{0,1}$ relevant for $K N$ and $\bar{K} N$ scattering,

$$
\begin{gathered}
C_{0}=2 d_{0}-2 d_{F}-d_{1}+4 b_{F}-4 b_{0}+\frac{D}{3 M_{0}}(3 F-D), \\
C_{1}=2 d_{D}+2 d_{0}+d_{1}-4 b_{0}-4 b_{D}-\frac{D^{2}+3 F^{2}}{6 M_{0}} .
\end{gathered}
$$

The reason for the appearance of only two independent combinations $C_{0,1}$ is crossing symmetry which relates $K N$ and $\bar{K} N$-scattering (see eq.(14) below for the general crossing relation).
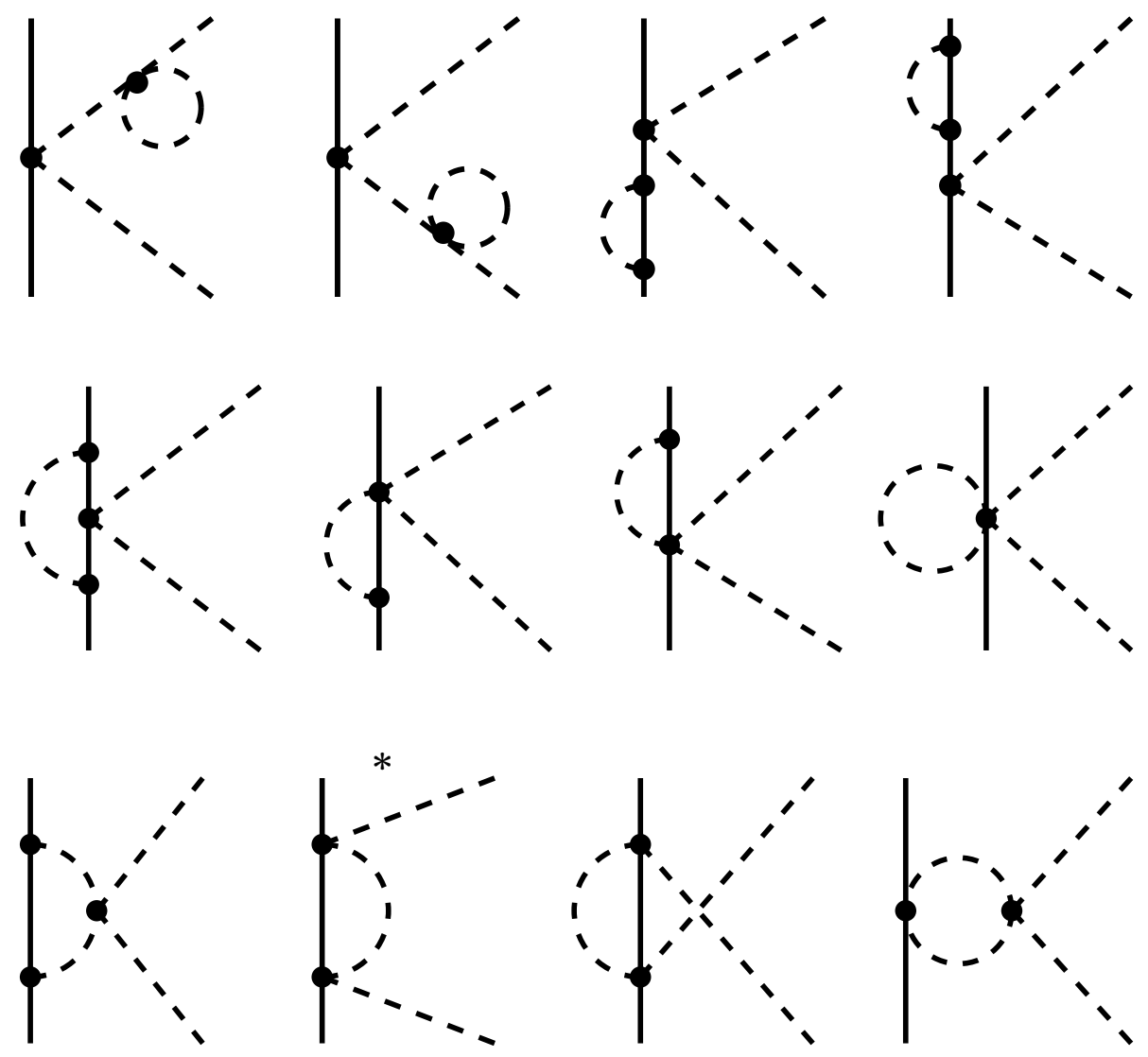

Fig. 1: Nonvanishing one-loop diagrams for meson-baryon scattering at threshold. Dashed lines represent Goldstone bosons $(\pi, K, \bar{K}, \eta)$ and full lines represent baryons $(N, \Lambda, \Sigma, \Xi)$. The nonvanishing imaginary parts of $T_{\bar{K}}^{(0,1)}$ come exclusively from the diagram marked by an asterisk.

At next-to-next-to-leading order $\mathcal{O}\left(q^{3}\right)$ one has the contribution from all one-loop graphs generated by the vertices of $\mathcal{L}_{\phi \phi}^{(2)}$ and $\mathcal{L}_{\phi B}^{(1)}$. In case of the threshold T-matrices the loop calculation simplifies considerably since all those diagrams in which the in- or out-going meson couples directly to the baryon line vanish identically $(\vec{\sigma} \cdot \vec{q}=0)$. The remaining set of non-vanishing oneloop diagrams is shown in Fig. 1. We use dimensional regularization and minimal subtraction 
to evaluate divergent loop integrals (for details see appendix B of ref.[8]). After renormalizing $f$ to $f_{K}$ in the leading order terms (as done already in eq.(6)) and summing up the contributions from all diagrams in Fig. 1 one finds the following renormalized one-loop chiral corrections to the $K N$ and $\bar{K} N$ threshold T-matrices,

$$
\begin{aligned}
& T_{K N}^{(0)}=\frac{m_{K}^{2}}{16 \pi^{2} f_{\pi}^{2} f_{K}^{2}}\left\{3 m_{K} \ln \frac{m_{\pi}}{\left|m_{K}\right|}+3 \sqrt{m_{K}^{2}-m_{\pi}^{2}} \ln \frac{m_{K}+\sqrt{m_{K}^{2}-m_{\pi}^{2}}}{m_{\pi}}\right. \\
& \left.+\pi(D-3 F)\left[(D+F) \frac{m_{\pi}^{2}}{m_{\eta}+m_{\pi}}+(7 D+3 F) \frac{m_{\eta}}{6}\right]\right\}, \\
& T_{K N}^{(1)}=\frac{m_{K}^{2}}{16 \pi^{2} f_{\pi}^{2} f_{K}^{2}}\left\{m_{K}\left(2 \ln \frac{m_{\pi}}{\lambda}+\ln \frac{\left|m_{K}\right|}{\lambda}+3 \ln \frac{m_{\eta}}{\lambda}-3\right)\right. \\
& +2 \sqrt{m_{K}^{2}-m_{\pi}^{2}} \ln \frac{m_{K}+\sqrt{m_{K}^{2}-m_{\pi}^{2}}}{m_{\pi}}-3 \sqrt{m_{\eta}^{2}-m_{K}^{2}} \arccos \frac{m_{K}}{m_{\eta}} \\
& \left.+\frac{\pi}{6}(3 F-D)\left[2(D+F) \frac{m_{\pi}^{2}}{m_{\eta}+m_{\pi}}+(D+5 F) m_{\eta}\right]\right\} \text {, } \\
& T_{\bar{K} N}^{(0)}=\frac{m_{K}^{2}}{32 \pi^{2} f_{\pi}^{2} f_{K}^{2}}\left\{3 m_{K}\left(3-\ln \frac{m_{\pi}}{\lambda}-2 \ln \frac{\left|m_{K}\right|}{\lambda}-3 \ln \frac{m_{\eta}}{\lambda}\right)\right. \\
& +3 \sqrt{m_{K}^{2}-m_{\pi}^{2}}\left(i \pi-\ln \frac{m_{K}+\sqrt{m_{K}^{2}-m_{\pi}^{2}}}{m_{\pi}}\right)-9 \sqrt{m_{\eta}^{2}-m_{K}^{2}} \arccos \frac{-m_{K}}{m_{\eta}} \\
& \left.+\pi(3 F-D)\left[2(D+F) \frac{m_{\pi}^{2}}{m_{\eta}+m_{\pi}}+(5 D+9 F) \frac{m_{\eta}}{3}\right]\right\} \\
& T_{\bar{K} N}^{(1)}=\frac{m_{K}^{2}}{32 \pi^{2} f_{\pi}^{2} f_{K}^{2}}\left\{m_{K}\left(3-5 \ln \frac{m_{\pi}}{\lambda}+2 \ln \frac{\left|m_{K}\right|}{\lambda}-3 \ln \frac{m_{\eta}}{\lambda}\right)\right. \\
& +5 \sqrt{m_{K}^{2}-m_{\pi}^{2}}\left(i \pi-\ln \frac{m_{K}+\sqrt{m_{K}^{2}-m_{\pi}^{2}}}{m_{\pi}}\right)-3 \sqrt{m_{\eta}^{2}-m_{K}^{2}} \arccos \frac{-m_{K}}{m_{\eta}} \\
& \left.+\frac{\pi}{3}(D-3 F)\left[2(D+F) \frac{m_{\pi}^{2}}{m_{\eta}+m_{\pi}}+(3 D-F) m_{\eta}\right]\right\}
\end{aligned}
$$

with $\lambda \sim 1 \mathrm{GeV}$ the scale parameter introduced in dimensional regularization. Note that we have used the symmetrical product $f_{\pi}^{2} f_{K}^{2}$ in the denominator of the prefactor in order to be more realistic on the rescattering process $\bar{K} N \rightarrow(\pi \Lambda, \pi \Sigma) \rightarrow \bar{K} N$ which generates imaginary parts. The corresponding diagram is the one marked by an asterisk in Fig. 1. The total sums of all (renormalized) one-loop corrections eqs. $(10,11,12,13)$ have remarkable properties which do not hold for the contributions from individual graphs. First, $m_{K}^{2}$ factors out. Secondly, the prefactors of chiral logarithms $\ln \left(m_{\pi, K, \eta} / \lambda\right)$ are independent of the axial vector coupling constants $D$ and $F$. Thirdly, the terms bilinear in $D$ and $F$ are finite, they carry an extra factor of $\pi$, and moreover $D-3 F$, which is proportional to the $\eta N N$-coupling constant, can be factored out of these contributions. The finite terms bilinear in $D$ and $F$ have been evaluated earlier in ref.[9]. Because of these properties there are only two chiral families of graphs in Fig. 1. Family 1 
is independent of $D$ and $F$ and it consists of the diagrams generated (exclusively) by mesonbaryon vertices with an even number of meson-lines. Family 2 on the other hand is bilinear in $D$ and $F$ and it comprises the diagrams generated (in addition) by meson-baryon vertices with an odd number of meson-lines. Note also that the loop contributions eqs. $(10,11,12,13)$ remain nonsingular in the $\mathrm{SU}(2)$ chiral limit $m_{\pi} \rightarrow 0$ and $m_{K, \eta}$ fixed. In addition to the contributions from one-loop graphs one has also further counterterm contributions at order $\mathcal{O}\left(q^{3}\right)$ which balance the scale dependence of the chiral logarithms $\ln \left(m_{\pi, K, \eta} / \lambda\right)$. In the case of threshold $\pi N$-scattering [2] the third order counterterm contribution has been estimated from resonance exchange and it was found to be much smaller than the chiral loop contribution. We assume that similar features hold for $K N$ and $\bar{K} N$-scattering. Note also that the low-energy constants $d_{D, F, 0,1}$ of the second order $\mathrm{SU}(3)$ chiral Lagrangian are not well determined at present. Let us finally mention the crossing symmetry relations which allow to calculate the $\bar{K} N$ threshold Tmatrices directly from the $K N$ threshold T-matrices by changing the sign of the kaon threshold energy $m_{K}$,

$$
T_{\bar{K} N}^{(0)}=\frac{1}{2}\left[3 T_{K N}^{(1)}-T_{K N}^{(0)}\right]_{m_{K} \rightarrow-m_{K}}, \quad T_{\bar{K} N}^{(1)}=\frac{1}{2}\left[T_{K N}^{(0)}+T_{K N}^{(1)}\right]_{m_{K} \rightarrow-m_{K}} .
$$

Obviously, these relations apply order by order in the chiral expansion.

As a further application of the full SU(3) scheme we reconsider the chiral expansion of the isospin-even and isospin-odd $\pi N$-scattering threshold T-matrices $T_{\pi N}^{ \pm}=4 \pi\left(1+m_{\pi} / M_{N}\right) a_{\pi N}^{ \pm}$. The contributions at order $\mathcal{O}(q)$ and $\mathcal{O}\left(q^{2}\right)$ appear selectively in the isospin-odd and isospin-even amplitude as,

$$
T_{\pi N}^{-}=\frac{m_{\pi}}{2 f_{\pi}^{2}}, \quad T_{\pi N}^{+}=\frac{m_{\pi}^{2}}{f_{\pi}^{2}}\left\{d_{D}+d_{F}+2 d_{0}-2 b_{D}-2 b_{F}-4 b_{0}-\frac{(D+F)^{2}}{4 M_{0}}\right\} .
$$

Here, we have again subsumed in $T_{\pi N}^{-}$already those loop corrections which renormalize $f$ to $f_{\pi}$, the physical pion decay constant. The remaining $(\pi, K, \eta)$-loop contributions at order $\mathcal{O}\left(q^{3}\right)$ generated by the diagrams in Fig. 1 are of the form,

$$
\begin{gathered}
T_{\pi N}^{+}=\frac{3 m_{\pi}^{2}}{64 \pi f_{\pi}^{4}}\left\{(D+F)^{2} m_{\pi}-2 \sqrt{m_{K}^{2}-m_{\pi}^{2}}-(D-3 F)^{2} \frac{m_{\eta}}{9}\right\}, \\
T_{\pi N}^{-}=\frac{m_{\pi}^{2}}{16 \pi^{2} f_{\pi}^{4}}\left\{m_{\pi}\left(\frac{3}{2}-2 \ln \frac{m_{\pi}}{\lambda}-\ln \frac{m_{K}}{\lambda}\right)-\sqrt{m_{K}^{2}-m_{\pi}^{2}} \arcsin \frac{m_{\pi}}{m_{K}}\right\} .
\end{gathered}
$$

Note that the kaon and eta-loop contributions to the isospin-even threshold T-matrix $T_{\pi N}^{+}$start out at order $m_{\pi}^{2}$ and therefore would have to be accounted for by the second order low-energy constants $c_{1,2,3}[2]$ in a reduction to $\mathrm{SU}(2)$. The isospin-odd threshold T-matrix $T_{\pi N}^{-}$on the other hand receives an additional small kaon-loop contribution proportional to $-1 / 2-\ln \left(m_{K} / \lambda\right)$ which will be viewed in a $\mathrm{SU}(2)$ reduction as a part of the third order low-energy constant $B^{r}(\lambda)$ (see ref.[2]).

Finally, we consider low-energy elastic $\pi \Lambda$-scattering in $\mathrm{SU}(3)$ baryon chiral perturbation theory. With only one total isospin-state and inherent crossing symmetry the $\pi \Lambda$-system is particularly simple. For the $\pi \Lambda$ threshold T-matrix $T_{\pi \Lambda}$ the leading order $\mathcal{O}(q)$ contribution vanishes. The second order $\mathcal{O}\left(q^{2}\right)$ contribution from the effective Lagrangian $\mathcal{L}_{\phi B}^{(2)}$ and the complete $\mathcal{O}\left(q^{3}\right)$ chiral loop corrections (see Fig. 1) read together,

$$
T_{\pi \Lambda}=\frac{2 m_{\pi}^{2}}{3 f_{\pi}^{2}}\left\{d_{D}+3 d_{0}-2 b_{D}-6 b_{0}-\frac{D^{2}}{2 M_{0}}\right\}+\frac{m_{\pi}^{2}}{16 \pi f_{\pi}^{4}}\left\{D^{2}\left(m_{\pi}-\frac{m_{\eta}}{3}\right)-3 \sqrt{m_{K}^{2}-m_{\pi}^{2}}\right\} .
$$


We will use this expression to estimate the $\pi \Lambda$ phase-shift at the $\Xi$-mass, a quantity which has recently received some interest $[10,11]$.

Next, we turn to the discussion of numerical results. We use for the meson masses $m_{\pi}=$ 139.57 MeV, $m_{K}=493.68 \mathrm{MeV}$ and $m_{\eta}=\sqrt{\left(4 m_{K}^{2}-m_{\pi}^{2}\right) / 3}=564.33 \mathrm{MeV}$ (the value implied by the GMO-relation which deviates only by $3.1 \%$ from the physical $\eta$-mass $m_{\eta}=547.3 \mathrm{MeV}$ $[12])$. The pion and kaon decay constants $f_{\pi}=92.4 \mathrm{MeV}$ and $f_{K}=113 \mathrm{MeV}$ [12] are wellknown and for the baryon axial vector coupling constants we use $D=0.8$ and $F=0.5$. This choice corresponds via the Goldberger-Treiman relation to a strong $\pi N N$-coupling constant of $g_{\pi N}=(D+F) M_{N} / f_{\pi}=13.2$ which is consistent with present empirical determinations [13]. The ratio $D / F=1.6$ agrees with the one extracted from semileptonic hyperon decays [14].

In order to make predictions and to determine remaining low-energy constants we do the following. The empirical value of the isospin-odd $\pi N$ threshold T-matrix $T_{\pi N}^{-}=(1.847 \pm$ $0.086) \mathrm{fm}[3]$ is reproduced at one-loop order (eqs. $(15,17)$ ) with the renormalization scale $\lambda$ set equal to $\lambda=0.95 \mathrm{GeV}$. We adopt then this value of $\lambda$ also for the $K N$ and $\bar{K} N$ threshold T-matrices. The combinations of low-energy constants $C_{0,1}$ are adjusted such that the empirical values of $T_{K N}^{(0,1)}$ (eq.(1)) are reproduced by their chiral expansions up to and including order $\mathcal{O}\left(q^{3}\right)$ (eqs. $(6,7,10,11))$. Inserting the resulting values $C_{0}=0.12 \mathrm{fm}=0.60 \mathrm{GeV}^{-1}$ and $C_{1}=0.41 \mathrm{fm}=$ $2.09 \mathrm{GeV}^{-1}$ into the formulas for $\bar{K} N$ threshold T-matrices (eqs. $(6,7,12,13)$ ) one predicts this way,

$$
T_{\bar{K} N}^{(0)}=(30.4+6.2 i) \mathrm{fm}, \quad T_{\bar{K} N}^{(1)}=(7.1+10.4 i) \mathrm{fm} .
$$

The complex-valued isospin-1 amplitude $T_{\bar{K} N}^{(1)}$ is in good agreement with the corresponding empirical value eq.(2), only its imaginary comes out about $10 \%$ too small. ${ }^{1}$ However, oneloop chiral perturbation theory fails completely in case of the isospin-0 amplitude $T_{\bar{K} N}^{(0)}$. The predicted real part has the right magnitude but the wrong positive sign and the imaginary is about a factor 2 too small. This should not come as a surprise since the isospin- $0 \bar{K} N$ s-wave channel is known to be completely dominated by the nearby subthreshold $\Lambda^{*}(1405)$-resonance. Only when employing non-perturbative methods $[16,17,18]$ one can generate the $\Lambda^{*}(1405)$ resonance as a quasi-bound $\bar{K} N$-state from the lowest order attractive chiral meson-baryon interaction in this channel. It is also not meaningful to add to the isospin- $0 \bar{K} N$ amplitude of one-loop chiral perturbation theory an explicit $\Lambda^{*}(1405)$-resonance contribution as done in [9].

The empirical value of the scattering length $a_{\bar{K} N}^{(0)}=(-1.70+0.68 i) \mathrm{fm}[5]$ and the presence of the subthreshold $\Lambda^{*}(1405)$-resonance are in fact intimately related with each other. In order to demonstrate this we show in Fig. 2 the real and imaginary part of the isospin- $0 \bar{K} N$ s-wave scattering amplitude in "unitarized" scattering length approximation [19],

$$
f_{\bar{K} N}^{(0)}(W)=\left[\left(a_{\bar{K} N}^{(0)}\right)^{-1}-i Q_{*}\right]^{-1}, \quad 2 Q_{*}=\sqrt{W^{2}-2\left(M_{N}^{2}+m_{K}^{2}\right)+\left(M_{N}^{2}-m_{K}^{2}\right)^{2} W^{-2}},
$$

as a function of the $\bar{K} N$ invariant mass $W$. The prominent resonance structure following already from the simple parametrization eq.(20) is clearly visible in Fig. 2. The position and slope of the zero-crossing of the real part $\operatorname{Re} f_{\bar{K} N}^{(0)}(W)$ suggest a resonance mass of $W_{0}=1416.4 \mathrm{MeV}$ and a width of $\Gamma=25.2 \mathrm{MeV}$. Such values of the mass and width are typically found for the $\Lambda^{*}$ (1405)-resonance in extrapolations below the $\bar{K} N$ threshold [12].

\footnotetext{
${ }^{1}$ Note that relativistic $1 / M_{0}$-corrections to the (complex-valued) one-loop amplitudes are considered as part of the higher order terms at $\mathcal{O}\left(q^{4}\right)$ in the heavy baryon formalism used here. A reevaluation of chiral corrections to $K N$ and $\bar{K} N$-scattering in a fully relativistic framework such as the one of ref.[15] can tell how important these corrections are for both the real and the imaginary part. Since the ratio $m_{K} / M_{0} \simeq 0.5$ is not that small, the effects could be sizeable.
} 




Fig. 2: The isospin-0 $\bar{K} N$ s-wave scattering amplitude in "unitarized" scattering length approximation versus the $\bar{K} N$ invariant mass $W$. The real part (imaginary part) of $f_{\bar{K} N}^{(0)}(W)$ is shown by the dashed (solid) line.

Let us now take a closer look at the chiral expansion of $T_{K N}^{(0,1)}$ and $T_{\bar{K} N}^{(0,1)}$. Numerically, one finds for the contributions at first, second and third chiral order: $T_{K N}^{(0)}=(0+2.29-1.87) \mathrm{fm}$, $T_{K N}^{(1)}=(-7.63+7.83-6.54) \mathrm{fm}, T_{\bar{K} N}^{(0)}=(11.44+10.59+8.35+6.23 i) \mathrm{fm}$ and $T_{\bar{K} N}^{(1)}=(3.81+$ $5.06-1.74+10.39 i) \mathrm{fm}$. One observes cancelations of large contributions at second and third chiral order, in particular for $T_{K N}^{(0,1)}$. This feature has also occurred in the chiral expansion of the baryon masses and magnetic moments performed to fourth order in ref.[7, 20]. Such cancelations of large terms seem to be generic for $\mathrm{SU}(3)$ baryon chiral perturbation theory calculations.

Finally, we want to use the present novel one-loop results to extract values of the s-wave lowenergy constants $d_{D, F, 0,1}$. The three chiral symmetry breaking parameters $b_{D, F, 0}$ and $M_{0}$ can be determined from the octet baryon masses and the $\pi N$ sigma-term $\sigma_{\pi N}(0)=45 \mathrm{MeV}$ (the central value of ref.[21]). Using the one-loop expressions given in chapter 6.1 of ref.[8] and inserting $f=f_{\pi}$ in $(\pi, \eta)$-loops and $f=f_{K}$ in kaon-loops one finds in a best fit $b_{D}=0.042 \mathrm{GeV}^{-1}$, $b_{F}=-0.557 \mathrm{GeV}^{-1}, b_{0}=-0.789 \mathrm{GeV}^{-1}$ and $M_{0}=918.4 \mathrm{MeV}$. With the additional piece of information given by the isospin-even $\pi N$ threshold T-matrix $T_{\pi N}^{+}=(-0.045 \pm 0.088) \mathrm{fm}[3]$ and the values $C_{0}=0.6 \mathrm{GeV}^{-1}$ and $C_{1}=2.1 \mathrm{GeV}^{-1}$ deduced from the $K N$ threshold T-matrices one obtains $d_{F}=-0.968 \mathrm{GeV}^{-1}, 2 d_{0}+d_{D}=-1.562 \mathrm{GeV}^{-1}$ and $d_{1}+d_{D}=1.150 \mathrm{GeV}^{-1}$. These numbers are comparable both in magnitude and sign with the ones of ref.[22]. There a large amount of pion and photon induced $(\eta, K)$-production data has been fitted in a coupled channel approach including s- and p-waves. If we furthermore assume that all second order low-energy constants do not exceed $1 \mathrm{GeV}^{-1}$ in magnitude (for which there is no known exception so far) we can make a prediction for the $\pi \Lambda$ s-wave phase-shift at the $\Xi$-mass. Using the scattering length approximation $\delta_{\pi \Lambda}=q_{\pi}^{c m} T_{\pi \Lambda}\left[4 \pi\left(1+m_{\pi} / M_{\Lambda}\right)\right]^{-1}$ with $q_{\pi}^{c m}=139 \mathrm{MeV}$ and the one-loop expression eq.(18) for $T_{\pi \Lambda}$ we obtain with $d_{0} \simeq-1.0 \mathrm{GeV}^{-1}$ [22] a $\pi \Lambda$ threshold T-matrix of $T_{\pi \Lambda} \simeq-1.0 \mathrm{fm}$ and a phase-shift at the $\Xi$-mass of $\delta_{\pi \Lambda} \simeq-2.8^{\circ}$. This is considerably smaller 
than the K-matrix result $\delta_{\pi \Lambda} \simeq-7^{\circ}$ of ref.[10]. The present perturbative one-loop result is however outside the very small range $0^{\circ} \leq \delta_{\pi \Lambda} \leq 1.1^{\circ}$ found in the relativistic chiral coupled channel approach of ref.[11].

In summary, we have calculated here in $\mathrm{SU}(3)$ heavy baryon chiral perturbation theory the threshold T-matrices for $K N, \bar{K} N, \pi N$ and $\pi \Lambda$ scattering to one-loop order. The kaonloop contribution to the isospin-odd amplitude $T_{\pi N}^{-}$is small. The complex-valued isospin-1 amplitude $T_{\bar{K} N}^{(1)}$ can be successfully predicted from the two $K N$ amplitudes $T_{K N}^{(0,1)}$. As expected perturbation theory fails in the isospin- $0 \bar{K} N$ channel which is completely dominated by the nearby subthreshold $\Lambda^{*}(1405)$-resonance. Cancelations of large terms at second and third order seem to be a generic feature of $\mathrm{SU}(3)$ baryon chiral perturbation theory calculations. All second order low-energy constants are of natural size and do not exceed $1 \mathrm{GeV}^{-1}$ in magnitude. At one-loop order we predict the $\pi \Lambda$ phase-shift at the $\Xi$-mass to be $\delta_{\pi \Lambda} \simeq-2.8^{\circ}$. In order to quantify the corrections to the present one-loop results fully relativistic calculations and higher order $\mathcal{O}\left(q^{4}\right)$ calculations in the heavy baryon framework of $\mathrm{SU}(3)$ chiral perturbation theory are needed.

\section{References}

[1] Y. Tomozawa, Nuovo Cimento 46A (1966) 707; S. Weinberg, Phys. Rev. Lett. 17 (1966) 616.

[2] V. Bernard, N. Kaiser and Ulf-G. Meißner, Phys. Lett. B309 (1993) 421; Phys. Rev. C52 (1995) 2185.

[3] H.Ch. Schröder et al., Phys. Lett. B469 (1999) 25.

[4] N. Fettes and Ulf-G. Meißner, Nucl. Phys. A676 (2000) 311; and refs. therein.

[5] A.D. Martin, Nucl. Phys. B179 (1981) 33.

[6] O. Dumbrajs et al., Nucl. Phys. B216 (1983) 277.

[7] B. Borasoy and Ulf-G. Meißner, Ann. Phys. (NY) 254 (1997) 192.

[8] V. Bernard, N. Kaiser and Ulf-G. Meißner, Int. J. Mod. Phys. E4 (1995) 193.

[9] C.H. Lee et al., Phys. Lett. B326 (1994) 14.

[10] J. Tandean, A.W. Thomas and G. Valencia, hep-ph/0011214.

[11] Ulf-G. Meißner and J.A. Oller, hep-ph/0011293.

[12] Particle Data Group, D.E. Groom et al., Eur. Phys. J. C15 (2000) 1.

[13] M.M. Pavan et al., Physica Scripta T87 (2000) 65.

[14] M. Bourquin et al., Z. Phys. C21 (1983) 27.

[15] T. Becher and H. Leutwyler, Eur. Phys. J. C9 (1999) 643; and refs. therein.

[16] N. Kaiser, P.B. Siegel and W. Weise, Nucl. Phys. A594 (1995) 325.

[17] E. Oset and A. Ramos, Nucl. Phys. A635 (1998) 99.

[18] J.A. Oller and Ulf-G. Meißner, Phys. Lett. B500 (2001) 263.

[19] N.M. Queen and G. Violini, "Dispersion theory in high energy physics" MacMillan Press, London, 1974, page 92.

[20] S. Steininger and Ulf-G. Meißner, Nucl. Phys. B499 (1997) 359.

[21] J. Gasser, H. Leutwyler and M.E. Sainio, Phys. Lett. B253 (1991) 252.

[22] J. Caro Ramon, N. Kaiser, S. Wetzel and W. Weise, Nucl. Phys. A672 (2000) 249. 